PROCEEDINGS OF THE

AMERICAN MATHEMATICAL SOCIETY

Volume 131, Number 8, Pages 2385-2391

S 0002-9939(03)06980-6

Article electronically published on February 26, 2003

\title{
THE FULL MARKOV-NEWMAN INEQUALITY FOR MÜNTZ POLYNOMIALS ON POSITIVE INTERVALS
}

\author{
DAVID BENKO, TAMÁS ERDÉLYI, AND JÓZSEF SZABADOS
}

(Communicated by Jonathan M. Borwein)

Abstract. For a function $f$ defined on an interval $[a, b]$ let

$$
\|f\|_{[a, b]}:=\sup \{|f(x)|: x \in[a, b]\} .
$$

The principal result of this paper is the following Markov-type inequality for Müntz polynomials.

Theorem. Let $n \geq 1$ be an integer. Let $\lambda_{0}, \lambda_{1}, \ldots, \lambda_{n}$ be $n+1$ distinct real numbers. Let $0<a<b$. Then

$$
\begin{aligned}
& \frac{1}{3} \sum_{j=0}^{n}\left|\lambda_{j}\right|+\frac{1}{4 \log (b / a)}(n-1)^{2} \leq \sup _{0 \neq Q} \frac{\left\|x Q^{\prime}(x)\right\|_{[a, b]}}{\|Q\|_{[a, b]}} \\
& \leq 11 \sum_{j=0}^{n}\left|\lambda_{j}\right|+\frac{128}{\log (b / a)}(n+1)^{2},
\end{aligned}
$$

where the supremum is taken for all $Q \in \operatorname{span}\left\{x^{\lambda_{0}}, x^{\lambda_{1}}, \ldots, x^{\lambda_{n}}\right\}$ (the span is the linear span over $\mathbb{R}$ ).

\section{INTRODUCTION AND NOTATION}

Let $\Lambda_{n}:=\left\{\lambda_{0}, \lambda_{1}, \ldots, \lambda_{n}\right\}$ be a set of $n+1$ distinct real numbers. The span of

$$
\left\{x^{\lambda_{0}}, x^{\lambda_{1}}, \ldots, x^{\lambda_{n}}\right\}
$$

over $\mathbb{R}$ will be denoted by

$$
M\left(\Lambda_{n}\right):=\operatorname{span}\left\{x^{\lambda_{0}}, x^{\lambda_{1}}, \ldots, x^{\lambda_{n}}\right\} .
$$

Elements of $M\left(\Lambda_{n}\right)$ are called Müntz polynomials of $n+1$ terms. For a function $f$ defined on an interval $[a, b]$ let

$$
\|f\|_{[a, b]}:=\sup \{|f(x)|: x \in[a, b]\}
$$

and let

$$
\|f\|_{L_{p}[a, b]}:=\left(\int_{a}^{b}|f(x)|^{p} d x\right)^{1 / p}, \quad p>0,
$$

Received by the editors March 2, 2002.

2000 Mathematics Subject Classification. Primary 41A17; Secondary 30B10, 26D15.

Key words and phrases. Müntz polynomials, exponential sums, Markov-type inequality, Newman's inequality.

The second author's research was supported, in part, by the NSF under Grant No. DMS0070826.

The third author's research was supported by OTKA Grant No. T32872. 
whenever the Lebesgue integral exists. Newman's beautiful inequality 4 , is an essentially sharp Markov-type inequality for $M\left(\Lambda_{n}\right)$ on $[0,1]$ in the case when each $\lambda_{j}$ is nonnegative.

Theorem 1.1 (Newman's Inequality). Let $\Lambda_{n}:=\left\{\lambda_{0}, \lambda_{1}, \ldots, \lambda_{n}\right\}$ be a set of $n+1$ distinct nonnegative numbers. Then

$$
\frac{2}{3} \sum_{j=0}^{n} \lambda_{j} \leq \sup _{0 \neq Q \in M\left(\Lambda_{n}\right)} \frac{\left\|x Q^{\prime}(x)\right\|_{[0,1]}}{\|Q\|_{[0,1]}} \leq 11 \sum_{j=0}^{n} \lambda_{j} .
$$

Note that the interval $[0,1]$ plays a special role in the study of Müntz polynomials. A linear transformation $y=\alpha x+\beta$ does not preserve membership in $M\left(\Lambda_{n}\right)$ in general (unless $\beta=0$ ), that is, $Q \in M\left(\Lambda_{n}\right)$ does not necessarily imply that $R(x):=Q(\alpha x+\beta) \in M\left(\Lambda_{n}\right)$. An analogue of Newman's inequality on $[a, b]$, $a>0$, cannot be obtained by a simple transformation. We can, however, prove the following result.

\section{NEW RESUlTS}

Theorem 2.1. Let $n \geq 1$ be an integer. Let $\Lambda_{n}:=\left\{\lambda_{0}, \lambda_{1}, \ldots, \lambda_{n}\right\}$ be a set of $n+1$ distinct real numbers. Let $0<a<b$. Then

$$
\begin{aligned}
\frac{1}{3} \sum_{j=0}^{n}\left|\lambda_{j}\right|+\frac{1}{4 \log (b / a)}(n-1)^{2} & \leq \sup _{0 \neq Q \in M\left(\Lambda_{n}\right)} \frac{\left\|x Q^{\prime}(x)\right\|_{[a, b]}}{\|Q\|_{[a, b]}} \\
& \leq 11 \sum_{j=0}^{n}\left|\lambda_{j}\right|+\frac{128}{\log (b / a)}(n+1)^{2} .
\end{aligned}
$$

Remarks 2.2. Of course, we can have $Q^{\prime}(x)$ instead of $x Q^{\prime}(x)$ in the above estimate, since an obvious corollary of the above theorem is

$$
\begin{aligned}
\frac{1}{3 b} \sum_{j=0}^{n}\left|\lambda_{j}\right|+\frac{1}{4 b \log (b / a)}(n-1)^{2} & \leq \sup _{0 \neq Q \in M\left(\Lambda_{n}\right)} \frac{\left\|Q^{\prime}\right\|_{[a, b]}}{\|Q\|_{[a, b]}} \\
& \leq \frac{11}{a} \sum_{j=0}^{n}\left|\lambda_{j}\right|+\frac{128}{a \log (b / a)}(n+1)^{2} .
\end{aligned}
$$

The reason we formulated Theorem 2.1 in the given form is that when $a \rightarrow 0$, we then obtain Theorem 1.1 (with worse absolute constants).

Theorem 2.1 was proved by P. Borwein and T. Erdélyi under the additonal assumptions that $\lambda_{j} \geq \delta j$ for each $j$ with a constant $\delta>0$ and with constants depending on $a, b$ and $\delta$ instead of the absolute constants (see [1] or [2, for instance).

The novelty of Theorem 2.1 is the fact that $\Lambda_{n}:=\left\{\lambda_{0}, \lambda_{1}, \ldots, \lambda_{n}\right\}$ is an arbitrary set of $n+1$ distinct real numbers; not even the nonnegativity of the exponents $\lambda_{j}$ is needed.

In the $L_{p}[a, b]$ norm $(p \geq 1)$ we can establish the following.

Theorem 2.3. Let $n \geq 1$ be an integer. Let $\Lambda_{n}:=\left\{\lambda_{0}, \lambda_{1}, \ldots, \lambda_{n}\right\}$ be a set of $n+1$ distinct real numbers. Let $0<a<b$ and $1 \leq p<\infty$. Then there is a positive constant $c_{1}(a, b)$ depending only on $a$ and $b$ such that

$$
\sup _{0 \neq P \in M\left(\Lambda_{n}\right)} \frac{\left\|P^{\prime}\right\|_{L_{p}[a, b]}}{\|P\|_{L_{p}[a, b]}} \leq c_{1}(a, b)\left(n^{2}+\sum_{j=0}^{n}\left|\lambda_{j}\right|\right) .
$$


Theorem 2.3 was proved by T. Erdélyi under the additonal assumptions that $\lambda_{j} \geq \delta j$ for each $j$ with a constant $\delta>0$ and with $c_{1}(a, b)$ replaced by $c_{1}(a, b, \delta)$; see [3]. The novelty of Theorem 2.3 is again the fact that $\Lambda_{n}:=\left\{\lambda_{0}, \lambda_{1}, \ldots, \lambda_{n}\right\}$ is an arbitrary set of $n+1$ distinct real numbers; not even the nonnegativity of the exponents $\lambda_{j}$ is needed.

\section{LeMmas}

The following comparison theorem for Müntz polynomials is proved in [1, E.4 f of Section 3.3].

Lemma 3.1 (A comparison theorem). Suppose

$$
\Lambda_{n}:=\left\{\lambda_{0}<\lambda_{1}<\cdots<\lambda_{n}\right\} \quad \text { and } \quad \Gamma_{n}:=\left\{\gamma_{0}<\gamma_{1}<\cdots<\gamma_{n}\right\},
$$

$\lambda_{n} \geq 0$, and $\lambda_{j} \leq \gamma_{j}$ for each $j=0,1, \ldots, n$. Let $0<a<b$. Then

$$
\max _{0 \neq Q \in M\left(\Lambda_{n}\right)} \frac{\left|Q^{\prime}(b)\right|}{\|Q\|_{[a, b]}} \leq \max _{0 \neq Q \in M\left(\Gamma_{n}\right)} \frac{\left|Q^{\prime}(b)\right|}{\|Q\|_{[a, b]}} .
$$

The following result is essentially proved by Saff and Varga [5]. They assume that $\Lambda:=\left(\lambda_{j}\right)_{j=0}^{\infty}$ is an increasing sequence of nonnegative integers and $\delta=1$ in the next lemma, however, this assumption can be easily dropped from their theorem; see [1, E.9 of Section 6.1]. In fact, their proof remains valid almost word for word; the modifications are straightforward.

Lemma 3.2 (The interval where the norm of a Müntz polynomial lives). Let

$$
\Lambda_{n}:=\left\{\lambda_{0}<\lambda_{1}<\cdots<\lambda_{n}\right\} \quad \text { and } \quad \lambda_{0} \geq 0 .
$$

Let $0 \neq P \in M\left(\Lambda_{n}\right)$ and $Q(x):=x^{k \delta} P(x)$, where $k$ is a nonnegative integer and $\delta$ is a positive real number. Let $\xi \in[0,1]$ be a point so that $|Q(\xi)|=\|Q\|_{[0,1]}$. Suppose $\lambda_{j} \geq \delta j$ for each $j$. Then

$$
\left(\frac{k}{k+n}\right)^{2 / \delta} \leq \xi
$$

\section{Proofs}

Proof of Theorem 2.1. First we prove the upper bound. Let $P \in M\left(\Lambda_{n}\right)$. We want to show that

$$
y\left|P^{\prime}(y)\right| \leq\left(11 \sum_{j=0}^{n}\left|\lambda_{j}\right|+\frac{128(n+1)^{2}}{\log (b / a)}\right)\|P\|_{[a, b]}
$$

for every $y \in[a, b]$. To this end we distinguish two cases. Without loss of generality we may assume that $\lambda_{k}=0$ for some $k$, otherwise we add the 0 exponent by changing $(n+1)$ to $n$.

Case 1. Let $y \in\left[(a b)^{1 / 2}, b\right]$. First we examine the subcase when $\lambda_{0}:=0$. That is, we have $0=\lambda_{0}<\lambda_{1}<\cdots<\lambda_{n}$. Let

$$
0<\delta:=\min \left\{1, \min _{1 \leq j \leq n} \frac{\lambda_{j}}{j}\right\} \leq 1
$$

Observe that the inequalities

$$
\lambda_{j} \geq \delta j, \quad j=1,2, \ldots, n,
$$


are satisfied. We define $Q(x):=x^{m n \delta} P(x)$, where with the choice $m:=\left\lfloor\frac{8 \log 2}{\delta \log (b / a)}\right\rfloor$, using the inequality $2^{-2 u} \leq 1-u(0 \leq u \leq 1 / 2)$ we have

$$
a=\sqrt{a b} \sqrt{\frac{a}{b}} \leq \sqrt{a b} 2^{-\frac{4}{\delta(m+1)}} \leq \sqrt{a b}\left(1-\frac{1}{m+1}\right)^{2 / \delta}=\sqrt{a b}\left(\frac{m}{m+1}\right)^{2 / \delta} .
$$

Scaling Newman's Inequality from $[0,1]$ to $[0, y]$, then using Lemma 3.2, we obtain

$$
\begin{aligned}
y\left|Q^{\prime}(y)\right| & \leq 11 \sum_{j=0}^{n}\left(\lambda_{j}+m n \delta\right)\|Q\|_{[0, y]} \\
& =11\left(\sum_{j=0}^{n} \lambda_{j}+m n(n+1) \delta\right)\|Q\|_{\left[y\left(\frac{m}{m+1}\right)^{2 / \delta}, y\right]} \\
& \leq 11\left(\sum_{j=0}^{n} \lambda_{j}+m n(n+1) \delta\right)\|Q\|_{[a, y]} .
\end{aligned}
$$

Hence

$$
\begin{aligned}
y\left|P^{\prime}(y)\right| & \leq\left|Q^{\prime}(y)\right| y^{1-m n \delta}+m n \delta|P(y)| \\
& \leq y^{-m n \delta} 11\left(\sum_{j=0}^{n} \lambda_{j}+m n(n+1) \delta\right)\|Q\|_{[a, y]}+m n \delta\|P\|_{[a, y]} \\
& \leq\left(11 \sum_{j=0}^{n} \lambda_{j}+m n(11 n+12) \delta\right)\|P\|_{[a, y]} \\
& \leq\left(11 \sum_{j=0}^{n} \lambda_{j}+\frac{128 n^{2}}{\log (b / a)}\right)\|P\|_{[a, b]} .
\end{aligned}
$$

This finishes the proof in Case 1 under the additional assumption $\lambda_{0}:=0$. Now we drop this additional assumption. Suppose

$$
\Lambda_{n}:=\left\{\lambda_{0}<\lambda_{1}<\cdots<\lambda_{n}\right\}
$$

and $\lambda_{k}=0$ for some $0 \leq k \leq n$. For a fixed $\varepsilon>0$ let

$$
\Gamma_{n, \varepsilon}:=\left\{\gamma_{0, \varepsilon}<\gamma_{1, \varepsilon}<\cdots<\gamma_{n, \varepsilon}\right\}
$$

with

$$
\gamma_{j, \varepsilon}:=(j-k) \varepsilon, \quad j=0,1,2, \ldots k,
$$

and

$$
\gamma_{j, \varepsilon}:=\lambda_{j}, \quad j=k+1, k+2, \ldots, n .
$$

If $\varepsilon>0$ is sufficiently small, then by Lemma 3.1 we have

$$
\max _{0 \neq Q \in M\left(\Lambda_{n}\right)} \frac{y\left|Q^{\prime}(y)\right|}{\|Q\|_{[a, y]}} \leq \max _{0 \neq Q_{\varepsilon} \in M\left(\Gamma_{n, \varepsilon}\right)} \frac{y\left|Q_{\varepsilon}^{\prime}(y)\right|}{\left\|Q_{\varepsilon}\right\|_{[a, y]}} .
$$

Let $Q_{\varepsilon} \in M\left(\Gamma_{n, \varepsilon}\right)$. Then $Q_{\varepsilon}$ is of the form

$$
Q_{\varepsilon}(x)=x^{-k \varepsilon} R_{\varepsilon}(x), \quad R_{\varepsilon} \in \operatorname{span}\left\{x^{\gamma_{0}+k \varepsilon}, x^{\gamma_{1}+k \varepsilon}, \ldots, x^{\gamma_{n}+k \varepsilon}\right\},
$$


where each $\gamma_{j}+k \varepsilon$ is nonnegative. Hence, using the upper bound of the theorem in the already proved case

$$
\lambda_{0}:=0, y \in\left[(a b)^{1 / 2}, b\right],
$$

we obtain

$$
y\left|R_{\varepsilon}^{\prime}(y)\right| \leq\left(11 \sum_{j=0}^{n}\left(\gamma_{j, \varepsilon}+k \varepsilon\right)+\frac{128 n^{2}}{\log (b / a)}\right)\left\|R_{\varepsilon}\right\|_{[a, y]} .
$$

Recalling (4.1), and taking the limit when $\varepsilon>0$ tends to 0 , we obtain

$$
\begin{aligned}
\max _{0 \neq Q \in M\left(\Lambda_{n}\right)} \frac{y\left|Q^{\prime}(y)\right|}{\|Q\|_{[a, y]}} & \leq \lim _{\varepsilon \rightarrow 0+} \max _{0 \neq Q_{\varepsilon} \in M\left(\Gamma_{n, \varepsilon}\right)} \frac{y\left|Q_{\varepsilon}^{\prime}(y)\right|}{\left\|Q_{\varepsilon}\right\|_{[a, y]}} \\
& \leq \lim _{\varepsilon \rightarrow 0+} 11 \sum_{j=0}^{n}\left(\gamma_{j, \varepsilon}+k \varepsilon\right)+\frac{128 n^{2}}{\log (b / a)} \\
& =11 \sum_{j=k+1}^{n} \lambda_{j}+\frac{128 n^{2}}{\log (b / a)} \\
& \leq 11 \sum_{j=0}^{n}\left|\lambda_{j}\right|+\frac{128 n^{2}}{\log (b / a)} .
\end{aligned}
$$

The proof of the upper bound of the theorem is now finished in Case 1.

Case 2. Let $y \in\left[a,(a b)^{1 / 2}\right]$. Suppose again that

$$
\Lambda_{n}:=\left\{\lambda_{0}<\lambda_{1}<\cdots<\lambda_{n}\right\}
$$

and $\lambda_{k}=0$ for some $0 \leq k \leq n$. Associated with $P \in M\left(\Lambda_{n}\right)$ let $\widetilde{P} \in M\left(\widetilde{\Lambda}_{n}\right)$ be defined by

$$
\begin{gathered}
\widetilde{P}(x):=P(a b / x), \\
\widetilde{\Lambda}_{n}:=\left\{\widetilde{\lambda}_{0}<\widetilde{\lambda}_{1}<\cdots<\widetilde{\lambda}_{n}\right\}:=\left\{-\lambda_{n}<-\lambda_{n-1}<\cdots<-\lambda_{0}\right\} .
\end{gathered}
$$

Using the upper bound of the theorem in the already proved Case 1 with $\widetilde{P} \in M\left(\widetilde{\Lambda}_{n}\right)$ and $\widetilde{y}=a b / y \in\left[(a b)^{1 / 2}, b\right]$, we obtain

$$
\begin{aligned}
y\left|P^{\prime}(y)\right| & =\left|\widetilde{P}^{\prime}(\widetilde{y})\right|(a b / y) \leq\left(11 \sum_{j=0}^{n}\left|\widetilde{\lambda}_{j}\right|+\frac{128(n+1)^{2}}{\log (b / a)}\right)\|\widetilde{P}\|_{[a, b]} \\
& =\left(11 \sum_{j=0}^{n}\left|\lambda_{j}\right|+\frac{128(n+1)^{2}}{\log (b / a)}\right)\|P\|_{[a, b]},
\end{aligned}
$$

and the proof is finished in Case 2 as well.

Now we show the lower bound of the theorem. Suppose

$$
\Lambda_{n}:=\left\{\lambda_{0}<\lambda_{1}<\cdots<\lambda_{n}\right\},
$$

and $0 \leq k \leq n$ is chosen so that $\lambda_{j}<0$ for all $j=1,2, \ldots, k$ and $\lambda_{j} \geq 0$ for all $j=k+1, k+2, \ldots, n$. Let

$$
\Lambda_{n}^{-}:=\left\{-\lambda_{k}<-\lambda_{k-1}<\cdots<-\lambda_{0}\right\}
$$


and

$$
\Lambda_{n}^{+}:=\left\{\lambda_{k+1}<\lambda_{k+2}<\cdots<\lambda_{n}\right\} .
$$

The lower bound of Theorem 1.1 (combined with a linear scaling, if necessary) shows the existence of a $Q \in M\left(\Lambda_{n}^{+}\right)$for which

$$
\left|Q^{\prime}(1)\right| \geq \frac{2}{3}\left(\sum_{j=k+1}^{n} \lambda_{j}\right)\|Q\|_{[0,1]} .
$$

Then $R(x):=Q(x / b) \in M\left(\Lambda_{n}\right)$ satisfies

$$
\begin{aligned}
\left\|x R^{\prime}(x)\right\|_{[a, b]} & \geq b\left|R^{\prime}(b)\right|=\left|Q^{\prime}(1)\right| \geq \frac{2}{3}\left(\sum_{j=k+1}^{n}\left|\lambda_{j}\right|\right)\|Q\|_{[0,1]} \\
& \geq \frac{2}{3}\left(\sum_{j=k+1}^{n}\left|\lambda_{j}\right|\right)\|R\|_{[a, b]} .
\end{aligned}
$$

Similarly, the lower bound of Theorem 1.1 (combined with a linear scaling, if necessary) shows the existence of a $Q \in M\left(\Lambda_{n}^{-}\right)$for which

$$
\left|Q^{\prime}(1)\right| \geq \frac{2}{3}\left(\sum_{j=0}^{k}\left(-\lambda_{j}\right)\right)\|Q\|_{[0,1]} .
$$

Then $R(x):=Q(a / x) \in M\left(\Lambda_{n}\right)$ satisfies

$$
\begin{aligned}
\left\|x R^{\prime}(x)\right\|_{[a, b]} & \geq a\left|R^{\prime}(a)\right|=\left|Q^{\prime}(1)\right| \geq \frac{2}{3}\left(\sum_{j=0}^{k}\left|\lambda_{j}\right|\right)\|Q\|_{[0,1]} \\
& \geq \frac{2}{3}\left(\sum_{j=0}^{k}\left|\lambda_{j}\right|\right)\|R\|_{[a, b]} .
\end{aligned}
$$

The two observations above already give

$$
\frac{1}{3} \sum_{j=0}^{n}\left|\lambda_{j}\right| \leq \sup _{0 \neq Q \in M\left(\Lambda_{n}\right)} \frac{\left\|x Q^{\prime}(x)\right\|_{[a, b]}}{\|Q\|_{[a, b]}} .
$$

To prove that

$$
\frac{(n-1)^{2}}{4 \log (b / a)} \leq \sup _{0 \neq \in M\left(\Lambda_{n}\right)} \frac{\left\|x Q^{\prime}(x)\right\|_{[a, b]}}{\|Q\|_{[a, b]}}
$$

we argue as follows. Let

$$
Q_{m, \varepsilon}(x):=T_{m}\left(\frac{2 x^{\varepsilon}}{b^{\varepsilon}-a^{\varepsilon}}-\frac{b^{\varepsilon}+a^{\varepsilon}}{b^{\varepsilon}-a^{\varepsilon}}\right) \in \operatorname{span}\left\{1, x^{\varepsilon}, x^{2 \varepsilon}, \ldots, x^{m \varepsilon}\right\},
$$

where $T_{m}(x)=\cos (m \arccos x), \quad x \in[-1,1]$, is the Chebyshev polynomial of degree $m$. Then

$$
\begin{aligned}
\frac{b\left|Q_{m, \varepsilon}^{\prime}(b)\right|}{\left\|Q_{m, \varepsilon}\right\|_{[a, b]}} & =\left|T_{m}^{\prime}(1)\right| \frac{2}{b^{\varepsilon}-a^{\varepsilon}} \varepsilon b^{\varepsilon} \\
& =\frac{2 m^{2}}{\varepsilon^{-1}\left(b^{\varepsilon}-1\right)-\varepsilon^{-1}\left(a^{\varepsilon}-1\right)} b^{\varepsilon} \underset{\varepsilon \rightarrow 0}{\longrightarrow} \frac{2 m^{2}}{\log b-\log a} .
\end{aligned}
$$


Now suppose, as before,

$$
\Lambda_{n}:=\left\{\lambda_{0}<\lambda_{1}<\cdots<\lambda_{n}\right\},
$$

and $0 \leq k \leq n$ is chosen so that $\lambda_{j}<0$ for all $j=1,2, \ldots, k$ and $\lambda_{j} \geq 0$ for all $j=k+1, k+2, \ldots, n$. Using Lemma 3.1 and (4.3), we obtain that for $k \leq n-1$ there is a

$$
Q \in \operatorname{span}\left\{x^{\lambda_{k+1}}, x^{\lambda_{k+2}}, \ldots, x^{\lambda_{n}}\right\}
$$

such that

$$
\frac{2(n-k-1)^{2}}{\log b-\log a} \leq \frac{b\left|Q^{\prime}(b)\right|}{\|Q\|_{[a, b]}} .
$$

Similarly, using Lemma 3.1 and (4.4), we obtain for $k \geq 0$ that there is an

$$
R \in \operatorname{span}\left\{x^{-\lambda_{0}}, x^{-\lambda_{1}}, \ldots, x^{-\lambda_{k}}\right\}
$$

such that

$$
\frac{2 k^{2}}{\log b-\log a} \leq \frac{b\left|R^{\prime}(b)\right|}{\|R\|_{[a, b]}}
$$

and hence for

$$
Q \in \operatorname{span}\left\{x^{\lambda_{0}}, x^{\lambda_{1}}, \ldots, x^{\lambda_{k}}\right\}
$$

defined by $Q(x):=R(a b / x)$ we have

$$
\frac{2 k^{2}}{\log b-\log a}=\frac{2 k^{2}}{\log b-\log a} \leq \frac{a\left|Q^{\prime}(a)\right|}{\|Q\|_{[a, b]}} .
$$

Now (4.2) follows from (4.4) and (4.5), and the proof of the lower bound of the theorem is finished.

Proof of Theorem 2.2. One can copy the proof in [3. by putting the upper bound of Theorem 1.1 in the appropriate place in the arguments. We omit the details.

\section{REFERENCES}

1. P. B. Borwein and T. Erdélyi, Polynomials and Polynomial Inequalities, Springer-Verlag, New York, 1995. MR 97e:41001

2. P. Borwein and T. Erdélyi, Newman's inequality for Müntz polynomials on positive intervals, J. Approx. Theory 85 (1996), 132-139. MR 97a:41007

3. T. Erdélyi, Markov- and Bernstein-type inequalities for Müntz polynomials and exponential sums in $L_{p}$, J. Approx. Theory 104 (2000), 142-152. MR 2001c:41014

4. D. J. Newman, Derivative bounds for Müntz polynomials, J. Approx. Theory 18 (1976), 360362. MR 55:3609

5. E. B. Saff and R. S. Varga, On lacunary incomplete polynomials, Math. Z. 177 (1981), 297314. MR 83a: 41008

Department of Mathematics, Texas A\&M University, College Station, Texas 77843

E-mail address: benko@math.tamu.edu

Department of Mathematics, Texas A\&M University, College Station, Texas 77843

E-mail address: terdelyi@math.tamu.edu

Alfréd Rényi Institute of Mathematics, P.O.B. 127, Budapest, Hungary, H-1364

E-mail address: szabados@renyi.hu 